\title{
Unsteady Aerodynamic Force Sensing from Strain Data
}

\author{
Chan-gi Pak* \\ NASA Armstrong Flight Research Center, Edwards Air Force Base, California 93523-0273 \\ DOI: $10.2514 / 1 . C 034140$
}

\begin{abstract}
A simple approach for computing unsteady aerodynamic forces from simulated measured strain data is proposed in this study. First, the deflection and slope of the structure are computed from the unsteady strain. Velocities and accelerations of the structure are computed using the autoregressive moving average model, online parameter estimator, low-pass filter, and a least-squares curve fitting method, together with analytical derivatives with respect to time. Finally, aerodynamic forces over the wing are computed using modal aerodynamic influence coefficient matrices, a rational function approximation, and a time-marching algorithm. A cantilevered rectangular wing is used to validate the simple approach. Unsteady aerodynamic forces as well as wing deflections, velocities, accelerations, and strains are computed using the CFL3D computational fluid dynamics code and the MSC/NASTRAN finite element analysis code; and these CFL3D/NASTRAN-based results are assumed as measured quantities. Computed deflections, velocities, accelerations, and unsteady aerodynamic forces are compared with the CFL3D/NASTRANbased results. Computed aerodynamic forces based on lifting-surface theory at subsonic speeds are in good agreement with the target aerodynamic forces generated using CFL3D code with the Euler equation. This research demonstrates the feasibility of obtaining induced drag and lift forces through the use of distributed sensor technology with measured strain data.
\end{abstract}

\section{Nomenclature}

$[A(s)] \quad=$ modal aerodynamic influence coefficient matrix in Laplace domain

$A_{i j} \quad=i$ th mode and $j$ th row coefficient for cosine function

$C_{i j} \quad=$ chord length at typical section

$\left[C_{j}\right]=j$ th aerodynamic lag term matrix in Roger's approximation at Mach $M_{i}$

$\left[D_{0}\right] \quad=$ constant term matrix in Roger's approximation

$\left[D_{1}\right]=$ linear term matrix in Roger's approximation

$\left[D_{2}\right] \quad=$ quadratic term matrix in Roger's approximation

$B_{i j}=i$ th mode and $j$ th row coefficient for sine function

$[E] \quad=$ state transition matrix

$[G] \quad=$ damping matrix

$[K] \quad=$ stiffness matrix

$k=$ discrete time

$L T=$ number of aerodynamic lag terms in Roger's approximation

$M \quad=$ Mach numbers

$[M] \quad=$ mass matrix

$\begin{array}{ll}m & =\text { number of reduced frequencies } \\ \{N(s)\} & =\text { orthonormalized aerodynamic force vector in }\end{array}$ Laplace domain

$\{N\}_{k}=$ orthonormalized aerodynamic force vector at discrete time $k$

$\mathrm{nm} \quad=$ number of modes

$\left\{\boldsymbol{Q}_{a}\right\}_{k}=$ generalized aerodynamic force vector at discrete time $k$

$q_{D} \quad=\quad$ dynamic pressure

$\{\boldsymbol{q}\}_{k}=$ generalized coordinates vector at discrete time $k$

$\left\{\boldsymbol{q}_{M}\right\}_{k}=$ master degree-of-freedom vector at discrete time $k$

$\left\{\boldsymbol{q}_{M e}(t)\right\}=$ measured master degree-of-freedom vector at continuous time $t$

$\left\{\boldsymbol{q}_{M e}\right\}_{k}=$ measured master degree-of-freedom vector at discrete time $k$

$\left\{\boldsymbol{q}_{S}\right\}_{k}=$ slave degree-of-freedom vector at discrete time $k$

$\begin{array}{lll}\left\{\tilde{\boldsymbol{q}}_{M e}\right\} & =\text { static equilibrium position of measured master } \\ s & =\text { Lapree-of-freedom vector } \\ T_{a} & =\text { time step for time-marching algorithm } \\ U & =\text { far-field air speed } \\ \{\boldsymbol{X}\}_{k} & =\text { state vector at discrete time } k \\ \epsilon_{\max } & =\text { local maximum unsteady strain } \\ \epsilon_{\mathrm{rms}} & =\text { root-mean-squared level of strain } \\ \{\boldsymbol{\epsilon}\}_{k} & =\text { strain vector at discrete time } k \\ \zeta_{i} & =\text { ith equivalent viscous damping factor } \\ n_{\mathrm{rms}} & =\text { root-mean-squared level of noise } \\ \{\boldsymbol{\eta}(s)\} & =\text { orthonormalized coordinates vector in Laplace } \\ & & \text { domain } \\ \{\boldsymbol{\eta}\}_{k} & =\text { orthonormalized coordinates vector at discrete } \\ & & \text { time } k \\ \kappa_{p} & =\text { reduced frequencies }\left(\equiv\left(\omega_{p} C / 2 U\right)\right), \text { where } p \text { is } \\ & =\text { equal to } 1,2, \ldots, m \\ \sigma_{i} & =\text { ith damping factor } \\ {[\Phi]} & =\text { eigenmatrix } \\ {\left[\Phi_{M}\right]} & =\text { eigenmatrix corresponds to master degrees of } \\ & \text { freedom } \\ {\left[\Phi_{S}\right]} & =\text { eigenmatrix corresponds to slave degrees of freedom } \\ \Omega_{j} & =\text { lag frequencies, where } j \text { is equal to } 1,2, \ldots, L T \\ \omega_{d i} & =\text { ith aeroelastic damped frequency } \\ \omega_{n i} & =\text { ith natural frequency of a structure } \\ \omega_{p} & =\text { frequencies for computing aerodynamic influence } \\ {[*]^{T}} & =\text { coefficient matrices } \\ {[*]^{-1}} & =\text { inverse of a matrix }[*] \\ \{*\} & =\text { velocity vector of }\{*\} \\ \{*\} & =\text { acceleration vector of }\{*\}\end{array}$

\section{Introduction}

$\mathbf{R}$ EDUCING fuel consumption for modern aircraft is a goal of the National Aeronautics and Space Administration (NASA) Aeronautics Research Mission Directorate. This goal can be accomplished by reducing airframe weight and aerodynamic drag; however, reductions in both for a civil transport aircraft is a challenge that may require extensive design changes for optimization and/or active controls. In general, the same percentage of weight and drag reductions can have a similar effect on fuel savings of a transport aircraft [1].

Real-time measurement of aerodynamic drag force in flight is an essential element for implementing an active drag control technique. Two major sources of aerodynamic drag on a business jet and a 
long-haul transport aircraft at cruise speed are viscous drag and induced drag, which are approximately $48-53 \%$ (one-half) and 21-38\% (one-third) of the total aerodynamic drag [1]. Moreover, induced drag comprises more than $90 \%$ of the total drag during takeoff for a typical transport aircraft [2].

Traditionally, lift forces over the wing are measured using a pressure gauge; however, the conventional pressure gauge with its associated tubing and cabling can create weight and space limitation challenges, and pressure data are available only at the discrete location of the gauge. A new method to measure lift forces is needed in order to overcome the weight and bulk associated with conventional pressure gauges. Development of lightweight distributed sensors is a critical technology that can allow continuous monitoring of aerodynamic surface shape, dynamic loading, and active control of flexible motion and drag.

Flexible and lightweight optical fibers not only revolutionized telecommunications but also altered the sensing world. Optical fibers can be used as fiber-optic sensors to measure strain and temperature [3]. Fiber-optic sensors have been developed to measure colocated strain simultaneously with very high accuracy using fiber Bragg gratings (FBGs) [3]. Specifically, the fiber-optic strain sensor (FOSS) uses a series of $F \bar{B} G$ s to obtain measurements at intervals as small as every half-inch [4] along a fiber and at frequencies of several kilohertz [5]. The ability of FBGs to operate at such high frequencies makes them an ideal choice for both static and dynamic aerospace applications. The methodology of optically measuring aerodynamic forces described by Liu et al. [6] is developed based on beam deformation theory. A two-camera videogrammetric system is used for optical deformation measurements. The data reduction models for extracting the normal force and pitching moment use either the local displacement and slope change or the global beam deformation profile.

The availability of wing deflections, velocities, and accelerations at all element grid points across the structural finite element (FE) model $[\underline{7}, \underline{8}]$ will allow engineers to undertake more accurate real-time analyses of both internal elastic and inertial forces, as well as external aerodynamic forces, at any point on the structure. These force values over the entire surface of a structure may also find application in structural health monitoring, active flexible motion control, and active drag reduction.

This study focuses on the computation of unsteady aerodynamic forces over an entire three-dimensional structure based on measured strain information. First, deformations of the entire three-dimensional structure are obtained using the two-step approach introduced by Pak [7]. Next, velocities and accelerations are computed using an autoregressive moving average (ARMA) model, online parameter estimator [9], low-pass filter, and a least-squares curve fitting method [10], together with analytical derivatives with respect to time. The unsteady aerodynamic forces are computed from structural deflections, velocities, and accelerations along with linear lifting-surface-based modal aerodynamic influence coefficient (AIC) matrices and a rational function approximation (RFA).

\section{Mathematical Background}

In this study, external unsteady aerodynamic forces are computed from measured strain data. Simulated strain data using CFL3D [11]/ MSC/NASTRAN [12] code will be assumed as measured strain data. In the first section, deflections and slopes of an entire structure are computed from measured strain through the use of the two-step approach [7]. Velocities and accelerations of the structure are computed in the second section using analytical derivatives with respect to time. In the last section, unsteady aerodynamic forces are computed in the time domain using the time-marching algorithm [13].

\section{A. Computation of Wing Deflection from Measured Strain}

Consider the following structural dynamic governing equations of motion as shown in Eq. (1):

$$
[M]\{\ddot{\boldsymbol{q}}\}_{k}+[G]\{\dot{\boldsymbol{q}}\}_{k}+[K]\{\boldsymbol{q}\}_{k}=\left\{\boldsymbol{Q}_{a}\right\}_{k}
$$

where $[M],[G]$, and $[K]$ are mass, damping, and stiffness matrices, respectively; and $\{\boldsymbol{q}\}_{k}$ and $\left\{\boldsymbol{Q}_{a}\right\}_{k}$ are the generalized coordinates and aerodynamic force vectors at discrete time $k$, respectively.

Out-of-plane deflections along FOSSs can be computed from measured unsteady strain data $\{\boldsymbol{\epsilon}\}_{k}$ using a piecewise least-squares method, an Akima spline, and a linear assumption, as described in the two-step approach [7]. These computed deflections along the fibers are combined with an FE model of the structure in order to interpolate and extrapolate the deflection and slope of the entire structure through the use of the system equivalent reduction and expansion process (SEREP) [14]. All of the degrees of freedom (DOFs) in the FE model can be rearranged, as shown in Eq. (2):

$$
\{\boldsymbol{q}\}_{k}=\left\{\begin{array}{c}
\boldsymbol{q}_{M} \\
\boldsymbol{q}_{S}
\end{array}\right\}_{k}=[\Phi]\{\boldsymbol{\eta}\}_{k}=\left[\begin{array}{c}
\Phi_{M} \\
\Phi_{S}
\end{array}\right]\{\boldsymbol{\eta}\}_{k}
$$

where $\left\{\boldsymbol{q}_{M}\right\}_{k}$ is the master DOF at discrete time $k$. In this approach, deflections along the FOSS computed from the first step of the twostep approach [7] are defined as the master DOF. The remaining deflections and slopes over all of the structure are defined as slave DOFs at discrete time $k,\left\{\boldsymbol{q}_{S}\right\}_{k}$. In Eq. (2), matrices $\left[\Phi_{M}\right]$ and $\left[\Phi_{S}\right]$ are eigenmatrices corresponding to master and slave DOFs, respectively; and $\{\boldsymbol{\eta}\}_{k}$ is the orthonormalized coordinates vector at discrete time $k$. Therefore, Eqs. (ㄱ) and (ㅁ) are derived from Eq. (2) :

$$
\begin{aligned}
\left\{\boldsymbol{q}_{M}\right\}_{k} & =\left[\Phi_{M}\right]\{\boldsymbol{\eta}\}_{k} \\
\left\{\boldsymbol{q}_{S}\right\}_{k} & =\left[\Phi_{S}\right]\{\boldsymbol{\eta}\}_{k}
\end{aligned}
$$

In Eq. (3), changing the master DOF at discrete time $k\left\{\boldsymbol{q}_{M}\right\}_{k}$ to the corresponding measured value $\left\{\boldsymbol{q}_{M e}\right\}_{k}$, along the FOSS gives Eq. (ㅁ):

$$
\left\{\boldsymbol{q}_{M e}\right\}_{k}=\left[\Phi_{M}\right]\{\boldsymbol{\eta}\}_{k}
$$

where $\left\{\boldsymbol{q}_{M e}\right\}_{k}$ is obtained from the first step of the two-step approach [7]. Premultiplying $\left[\Phi_{M}\right]^{T}$ to Eq. (5) with matrix inversion gives Eq. (6) for computing the orthonormalized coordinates vector at discrete time $k$ :

$$
\{\boldsymbol{\eta}\}_{k}=\left(\left[\Phi_{M}\right]^{T}\left[\Phi_{M}\right]\right)^{-1}\left[\Phi_{M}\right]^{T}\left\{\boldsymbol{q}_{M e}\right\}_{k}
$$

and the generalized coordinates vector $\{\boldsymbol{q}\}_{k}$ of Eq. (7) is obtained from substituting Eq. (ㅁ) into Eq. (2)):

$$
\left\{\boldsymbol{q}_{k}=\left[\begin{array}{c}
\Phi_{M} \\
\Phi_{S}
\end{array}\right]\left(\left[\Phi_{M}\right]^{T}\left[\Phi_{M}\right]\right)^{-1}\left[\Phi_{M}\right]^{T}\left\{\boldsymbol{q}_{M e}\right\}_{k}\right.
$$

\section{B. Computation of Velocity and Acceleration from Computed Wing Deflection}

A simple harmonic motion assumption for the computation of wing acceleration works with undamped free vibration problems [8], but this assumption cannot handle the heavy damping issues associated with aeroelastic oscillation problems. Also, the orthonormalized coordinate vector $\{\boldsymbol{\eta}\}_{k}$ used for the computation of velocities in [ $[\underline{]}]$ is not fully decoupled because of coupling between structural dynamics and unsteady aerodynamics.

A new approach for the computations of aeroelastic velocity and acceleration is proposed in this study. Velocity and acceleration vectors at each sensor location at discrete time $k,\left\{\dot{\boldsymbol{q}}_{M e}\right\}_{k}$ and $\left\{\ddot{\boldsymbol{q}}_{M e}\right\}_{k}$, of an aeroelastic structural motion are computed using Eq. ( $\underline{8})$ together with analytical derivatives with respect to time:

$$
\left\{\boldsymbol{q}_{M e}(t)\right\}=\left\{\tilde{\boldsymbol{q}}_{M e}\right\}+\left\{\sum_{i=1}^{\mathrm{nm}} \boldsymbol{e}^{-\sigma_{i} t}\left[A_{i j} \cos \left(\boldsymbol{\omega}_{d i} \boldsymbol{t}\right)+\boldsymbol{B}_{i j} \sin \left(\boldsymbol{\omega}_{d i} t\right)\right]\right\}
$$


where $\sigma_{i}\left(=\zeta_{i} \omega_{n i}\right)$ and $\omega_{d i}$ are the $i$ th damping factor and damped frequency, respectively; and $\mathrm{nm}$ is the number of modes. A vector $\left\{\tilde{\boldsymbol{q}}_{M e}\right\}$ represents the static equilibrium position of the unsteady wing motion. The coefficients $A_{i j}$ and $B_{i j}, j=1,2, \ldots, n$, for the $j$ th row element of the vector can be fitted using a least-squares curve fitting technique $[9,10]$. In this study, $\sigma_{i}$ and $\omega_{d i}$ are computed using an ARMA model, an online parameter estimator, and a sine Butterworth low-pass filter [15]. It should be noted in Eq. (8) that $\sigma_{i}$ and $\omega_{d i}$ are estimated; therefore, the least-squares curve fitting in this study is based on a linear fitting. From Eq. (ㅇ), the velocity and acceleration are computed using analytical derivatives with respect to time $t$. The FE model is not used for the computation of $\left\{\boldsymbol{q}_{M e}\right\}_{k},\left\{\dot{\boldsymbol{q}}_{M e}\right\}_{k}$, and $\left\{\ddot{\boldsymbol{q}}_{M e}\right\}_{k}$.

Velocity and acceleration vectors over the entire structure are also computed using Eqs. (9) and (10) (SEREP transformation):

$$
\begin{aligned}
\{\dot{\boldsymbol{q}}\}_{k} & =\left[\begin{array}{c}
\Phi_{M} \\
\Phi_{S}
\end{array}\right]\left(\left[\Phi_{M}\right]^{T}\left[\Phi_{M}\right]\right)^{-1}\left[\Phi_{M}\right]^{T}\left\{\dot{\boldsymbol{q}}_{M e}\right\}_{k} \\
\{\ddot{\boldsymbol{q}}\}_{k} & =\left[\begin{array}{c}
\Phi_{M} \\
\Phi_{S}
\end{array}\right]\left(\left[\Phi_{M}\right]^{T}\left[\Phi_{M}\right]\right)^{-1}\left[\Phi_{M}\right]^{T}\left\{\ddot{\boldsymbol{q}}_{M e}\right\}_{k}
\end{aligned}
$$

\section{Computation of Aerodynamic Force from Wing Deflection,}

\section{Velocity, and Acceleration}

First, modal AIC matrices are computed at Mach number $M$ and reduced frequencies $\kappa_{p}\left(\equiv\left(\omega_{p} C / 2 U\right), p=1,2, \ldots, m\right)$ using lifting-surface theory:

$$
\left[A\left(\kappa_{1}\right)\right], \quad\left[A\left(\kappa_{2}\right)\right], \ldots,\left[A\left(\kappa_{m}\right)\right]
$$

where $C$ is the chord length at a typical section, and $U$ is a far-field airspeed. These modal AIC matrices can be approximated with respect to frequency and Laplace variable $s$ using an RFA. In this study, Roger's approximation [Eq. (11)] is selected for the RFA:

$$
[A(s)]=\left[D_{0}\right]+s\left[D_{1}\right]+s^{2}\left[D_{2}\right]+\sum_{j=1}^{L T} \frac{s\left[C_{j}\right]}{s+\Omega_{j}}
$$

Substituting Eq. (2) into Eq. (1) and premultiplying $[\Phi]^{T}$ yields Eq. (12):

$$
\begin{gathered}
{[\Phi]^{T}[M][\Phi]\{\ddot{\boldsymbol{\eta}}\}_{k}+[\Phi]^{T}[G][\Phi]\{\dot{\boldsymbol{\eta}}\}_{k}+[\Phi]^{T}[K][\Phi]\{\boldsymbol{\eta}\}_{k}} \\
=[\Phi]^{T}\left\{\boldsymbol{Q}_{a}\right\}_{k}=\{\boldsymbol{N}\}_{k}
\end{gathered}
$$

The orthonormalized aerodynamic force vector $\{N(s)\}$ in the Laplace domain is in Eq. (13):

$$
\begin{aligned}
&\{\boldsymbol{N}(\boldsymbol{s})\}=\boldsymbol{q}_{D}[A(\boldsymbol{s})]\{\boldsymbol{\eta}(\boldsymbol{s})\} \\
&=\boldsymbol{q}_{D}\left(\left[D_{0}\right]\{\boldsymbol{\eta}(\boldsymbol{s})\}+\boldsymbol{s}\left[D_{1}\right]\{\boldsymbol{\eta}(\boldsymbol{s})\}+\boldsymbol{s}^{2}\left[D_{2}\right]\{\boldsymbol{\eta}(\boldsymbol{s})\}\right. \\
&\left.+\sum_{j=1}^{L T} \frac{\boldsymbol{s}\left[C_{j}\right]\{\boldsymbol{\eta}(\boldsymbol{s})\}}{\boldsymbol{s}+\Omega_{j}}\right)
\end{aligned}
$$

The time-marching algorithm for the computation of the orthonormalized aerodynamic force at discrete time $k$ can be summarized as follows [13] in Eqs. (14-21):

$$
\begin{array}{r}
\{\boldsymbol{N}\}_{k}=\boldsymbol{q}_{D}\left(\left[D_{0}\right]\{\boldsymbol{\eta}\}_{k}+\left[D_{1}\right]\{\dot{\boldsymbol{\eta}}\}_{k}+\left[D_{2}\right]\{\ddot{\boldsymbol{\eta}}\}_{k}+[C]\{\boldsymbol{x}\}_{k}\right) \\
\{\boldsymbol{X}\}_{k}=[E]\{\boldsymbol{X}\}_{k-1}+[\boldsymbol{\theta}][\boldsymbol{B}] \frac{\{\dot{\boldsymbol{\eta}}\}_{k}+\{\dot{\boldsymbol{\eta}}\}_{k-1}}{2}
\end{array}
$$

where

$$
\begin{gathered}
{[E]=\boldsymbol{e}^{[A] T_{a}}} \\
{[\boldsymbol{\theta}]=\int_{0}^{T_{a}} \boldsymbol{e}^{[A]\left(T_{a}-\tau\right)} \mathrm{d} \boldsymbol{\tau}} \\
{[A]=\left[\begin{array}{cccc}
-\Omega_{1} \boldsymbol{I} & 0 & \ldots & 0 \\
0 & -\Omega_{2} \boldsymbol{I} & \ldots & 0 \\
\vdots & \vdots & \ddots & \vdots \\
0 & 0 & \ldots & -\Omega_{L T} \boldsymbol{I}
\end{array}\right]}
\end{gathered}
$$

$$
\begin{gathered}
{[\boldsymbol{B}]=\left[\begin{array}{c}
\boldsymbol{I} \\
\boldsymbol{I} \\
\vdots \\
\boldsymbol{I}
\end{array}\right]} \\
{[C]=\left[C_{1} C_{2}, \ldots, C_{L T}\right]}
\end{gathered}
$$

$$
\{\boldsymbol{X}\}_{k}=\left\{\begin{array}{c}
\boldsymbol{x}_{1} \\
\boldsymbol{x}_{2} \\
\vdots \\
\boldsymbol{x}_{L T}
\end{array}\right\}_{k}
$$

and $T_{a}$ is a sampling time. Orthonormalized coordinate vectors $\{\boldsymbol{\eta}\}_{k}$, $\{\dot{\boldsymbol{\eta}}\}_{k}$, and $\{\ddot{\boldsymbol{\eta}}\}_{k}$ are computed from Eqs. (22-24):

$$
\{\boldsymbol{\eta}\}_{k}=\left(\left[\Phi_{M}\right]^{T}\left[\Phi_{M}\right]\right)^{-1}\left[\Phi_{M}\right]^{T}\left\{\boldsymbol{q}_{M e}\right\}_{k}
$$

$$
\{\dot{\boldsymbol{\eta}}\}_{k}=\left(\left[\Phi_{M}\right]^{T}\left[\Phi_{M}\right]\right)^{-1}\left[\Phi_{M}\right]^{T}\left\{\dot{\boldsymbol{q}}_{M e}\right\}_{k}
$$

$$
\{\ddot{\boldsymbol{\eta}}\}_{k}=\left(\left[\Phi_{M}\right]^{T}\left[\Phi_{M}\right]\right)^{-1}\left[\Phi_{M}\right]^{T}\left\{\ddot{\boldsymbol{q}}_{M e}\right\}_{k}
$$

From Eq. (12), the generalized aerodynamic force vector at discrete time $k,\left\{\boldsymbol{Q}_{a}\right\}_{k}$, is shown in Eq. (25):

$$
\left\{\boldsymbol{Q}_{a}\right\}_{k}=\left([\Phi]^{T}\right)^{-1}\{\boldsymbol{N}\}_{k}
$$

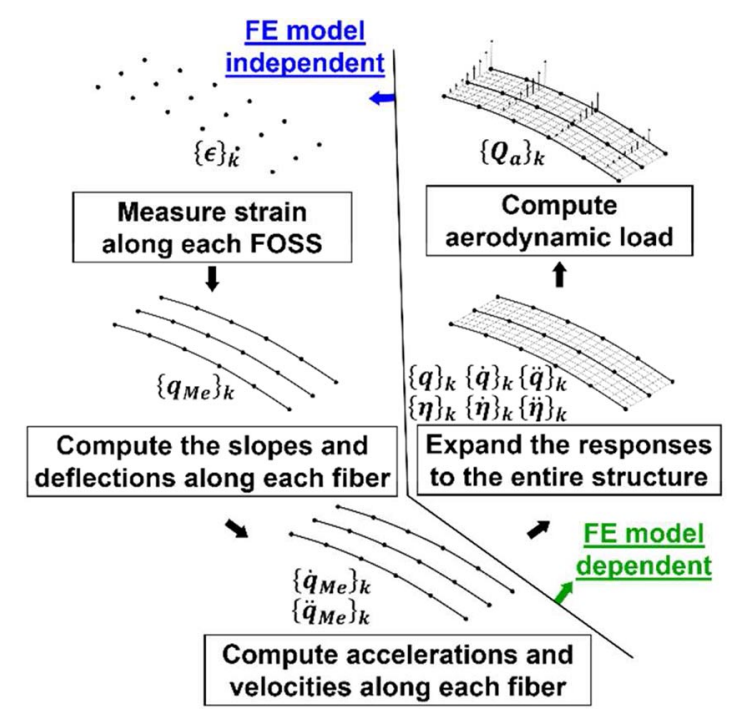

Fig. 1 Steps used to compute aerodynamic force from measured strain. 
A rectangular matrix $[\Phi]^{T}$ can be inverted using a singular-value decomposition technique. The steps used to compute aerodynamic force from measured strain are depicted in Fig. 1.

In general, aerodynamic force vectors from a lifting-surface theory are normal to the aerodynamic model configuration. Therefore, unsteady induced drag force as well as lateral force can be defined using the surface normal vector during the unsteady wing surface oscillation, as shown in Fig. 2.

\section{Summary of Computation of Aerodynamic Force from Strain}

The following matrices should be calculated before starting the computation of unsteady aerodynamic loads during flight:

1) The first matrices are modal AIC matrices and corresponding matrices from RFA: $\left[D_{0}\right],\left[D_{1}\right],\left[D_{2}\right],\left[C_{1} C_{2}, \ldots, C_{L T}\right],[E]$ in Eqs. (14) and (16), and $[\boldsymbol{\theta}][B]$ in Eq. (15). Matrices $[E]$ and $[\boldsymbol{\theta}][\boldsymbol{B}]$ are the function of aerodynamic lag frequencies for the Roger's approximation

2) The second matrices are the transformation matrices based on the SEREP approach and singular value decomposition: $\left(\left[\Phi_{M}\right]^{T}\left[\Phi_{M}\right]\right)^{-1}\left[\Phi_{M}\right]^{T}$ and $\left([\Phi]^{T}\right)^{-1}$.

Step 1) Collect unsteady strain $\{\boldsymbol{\epsilon}\}_{k}$.

Step 2) Compute wing deflection along the FOSS line, $\left\{\boldsymbol{q}_{M e}\right\}_{k}$, using the two-step approach.

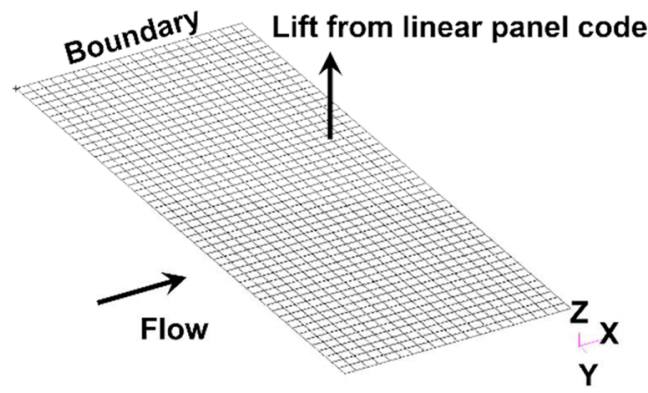

a) ZAERO aerodynamic model

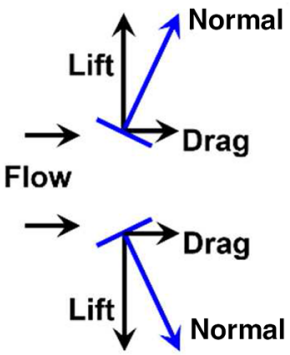

b) Side view of the unsteady wing motion

Fig. 2 Definition of the unsteady aerodynamic forces from a linear lifting-surface theory.

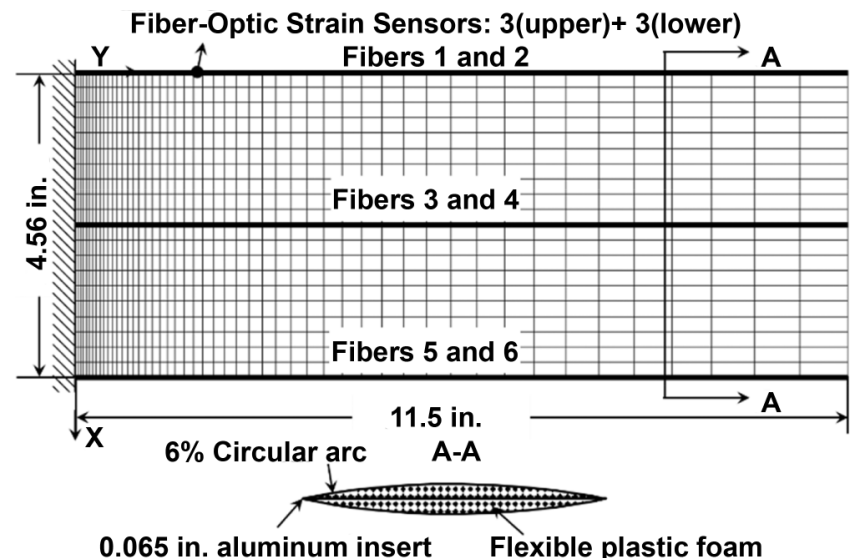

065 in aluminum insert Flexible plastic foam

Fig. 3 Cantilevered rectangular wing with six FOSSs.
Table 1 Detailed material properties of the cantilevered plate wing

\begin{tabular}{lc}
\hline \hline Properties of aluminum insert & Number \\
\hline Young's modulus $E$ & $9207766 \mathrm{psi}$ \\
Shear modulus $G$ & $3836570 \mathrm{psi}$ \\
Density & $0.1 \mathrm{lb} / \mathrm{in}^{3}$ \\
Total weight & $0.3806 \mathrm{lb}$ \\
Xcg & $2.28 \mathrm{in}$. \\
Ycg & $5.75 \mathrm{in}$. \\
Thickness & $0.065 \mathrm{in}$. \\
\hline \hline
\end{tabular}

Table 2 Measured and computed natural frequencies

\begin{tabular}{lccc}
\hline \hline Mode & Measured, $\mathrm{Hz}$ & Computed, $\mathrm{Hz}$ & Comment \\
\hline 1 & 14.29 & 14.29 & First bending \\
2 & 80.41 & 80.17 & First torsion \\
3 & 89.80 & 89.04 & Second bending \\
\hline \hline
\end{tabular}

Step 3) Compute wing velocity and acceleration along the FOSS line: $\left\{\dot{\boldsymbol{q}}_{M e}\right\}_{k}$ and $\left\{\ddot{\boldsymbol{q}}_{M e}\right\}_{k}$.

Step 4) Compute $\{\boldsymbol{\eta}\}_{k},\{\dot{\boldsymbol{\eta}}\}_{k}$, and $\{\ddot{\boldsymbol{\eta}}\}_{k}$ using Eqs. (22-24).

Step 5) Compute the orthonormalized aerodynamic force vector $\{N\}_{k}$ using Eqs. (14) and (15).

Step 6) Convert the orthonormalized aerodynamic force vector $\{N\}_{k}$ to the generalized aerodynamic force vector $\left\{\boldsymbol{Q}_{a}\right\}_{k}$ using Eq. (25). The $z$-directional load is the lift load.

Step 7) Compute induced drag and lateral forces using surface normal vectors together with the lift force in step 6 .

Steps 1 through 3 are the model independent procedures. On the other hand, steps 4 through 7 are dependent on the structural dynamic model $\Phi$ and the unsteady aerodynamic model, $\left[D_{0}\right],\left[D_{1}\right],\left[D_{2}\right]$, $\left[C_{1} C_{2}, \ldots, C_{L t}\right],[E]$, and $[\boldsymbol{\theta}][\boldsymbol{B}]$.

\section{Results and Discussions}

A cantilevered rectangular wing, shown in Fig. 3, was selected for the validation of the proposed approach. This wing, with $6 \%$ circular arc cross sections and an aspect ratio of 5.0, was built and tested at the NASA Langley Research Center (Hampton, Virginia) in 1959 [16]. The model had a uniform chord length of 4.56 in., a span length of $11.5 \mathrm{in}$., and a thickness of $0.065 \mathrm{in}$. of aluminum insert covered with flexible plastic foam (Fig. 3). The material properties of the aluminum insert were assigned a Young's modulus $E$ of 9.208 Msi (Msi $=1,000,000 \mathrm{psi}$ ); a shear modulus $G$ of $3.837 \mathrm{Msi}$; and a mass density of $0.1 \mathrm{lb} /$ in. $^{3}$. The shaped lumped weights were used to match the local cross-sectional weight distribution of the plastic foam. Therefore, the small lumped weights were used near the leading and trailing edges, and the large lumped weights were used near the midchord area. Detailed material properties are shown in Table 1. To represent the six FOSSs, the model was fit with 300 beam elements

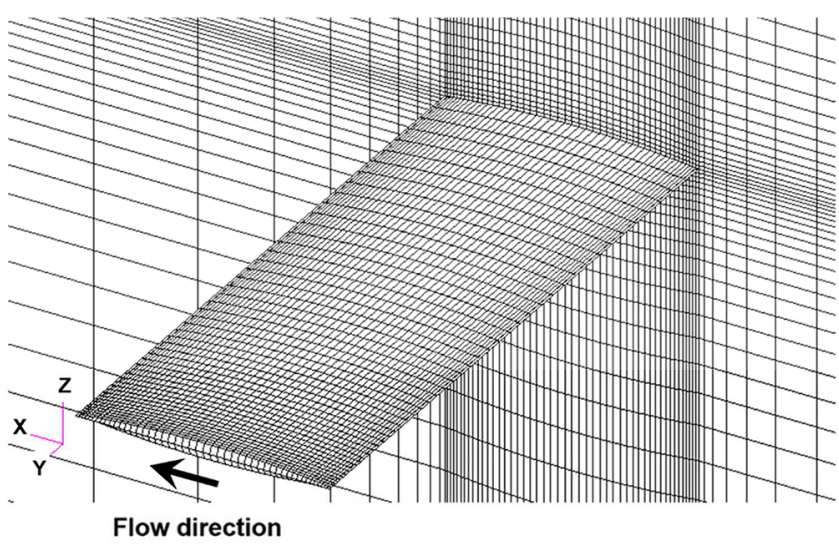

Fig. 4 CFD grid for CFL3D computations based on Euler grid. 


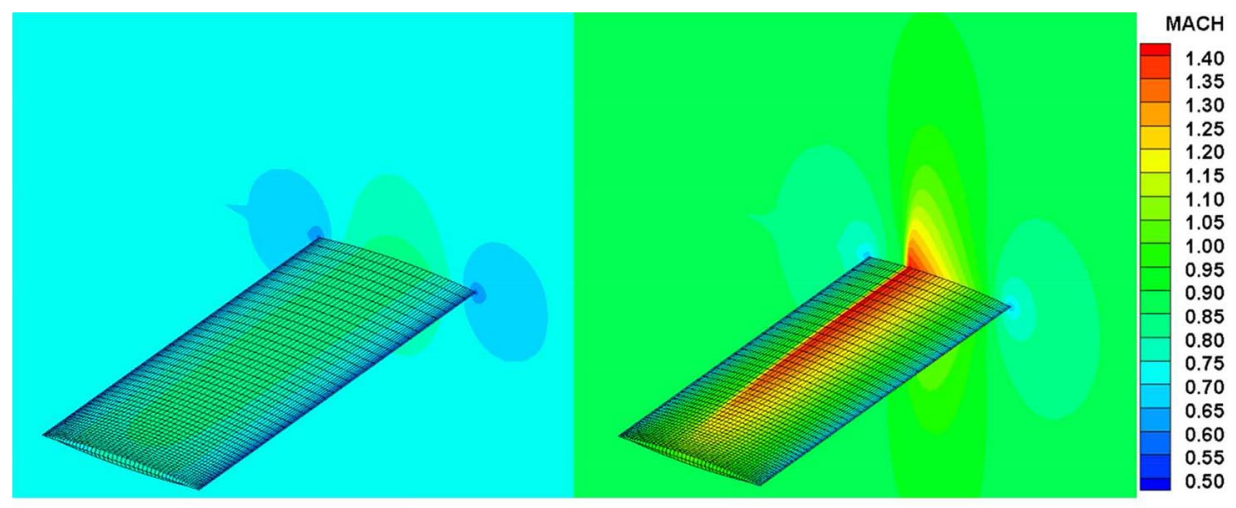

a) $\mathrm{M}=\mathbf{0 . 7 1 4}$

b) $\mathrm{M}=\mathbf{0 . 8 7 5}$

Fig. 5 Local Mach number distributions at Mach 0.714 and 0.875 .

(50 per each fiber) that computed the axial strain along the length of the wing. These six simulated FOSSs were assumed to be zero weight with zero stiffness (Fig. 3).

The frequencies and mode shapes of this cantilevered wing model are computed using the MSC/NASTRAN code [12]. Measured and computed natural frequencies are summarized in Table 2 . Unsteady aerodynamic forces as well as wing deflections and velocities are computed using the CFL3D code [11]. However, acceleration and unsteady strain data are not available from the CFL3D computation; therefore, the MSC/NASTRAN code is used to compute target acceleration and simulated measured strain data.

A computational fluid dynamics (CFD) grid configuration for the CFL3D computations based on the Euler grid is given in Fig. 4. The CFD grid is a multiblock $(97 \times 73 \times 57)$ grid with $\mathrm{H}-\mathrm{H}$ topology. The time-step size of the unsteady CFL3D computation is $0.000060515 \mathrm{~s}$, and a total of 10,240 time steps are used in this computation. The unsteady aerodynamic theory used in Sec. II.C is based on a linear lifting-surface theory: ZAERO code [17]. Therefore, a subsonic Mach number should be selected for the CFL3D computer simulation to minimize a nonlinear transonic effect. Local Mach number distributions under steady-state conditions with CFL3D computer simulations are given in Fig. $\underline{5}$. In this figure, local Mach number distributions at Mach 0.714 are high subsonic conditions. The maximum local Mach number reaches the 0.8-0.9 range near the center chord, as shown in Fig. 5a. Supersonic subregions are observed in the Mach 0.875 case (that is, transonic speed), as shown in Fig. 5b. Therefore, a Mach number of 0.714 with dynamic pressure of $1.4 \overline{55}$ psi is selected for the validation of the current approach. These CFD-based aerodynamic forces are assumed as the target forces in this study.

The MSC/NASTRAN code was used to calculate unsteady strains in this study, and these computed strains are considered as the measured strains. For the CFL3D computations, structural mode shapes should be provided at the CFD grid points. In this study, the structural grid points and the CFD grid points are connected using the interpolation elements ("RBE3 element" in MSC/NASTRAN terminology) instead of using a surface-splining technique. In the CFL3D code, unsteady aerodynamic force vectors are computed at the centroids of CFD cells. Therefore, a splining between structural

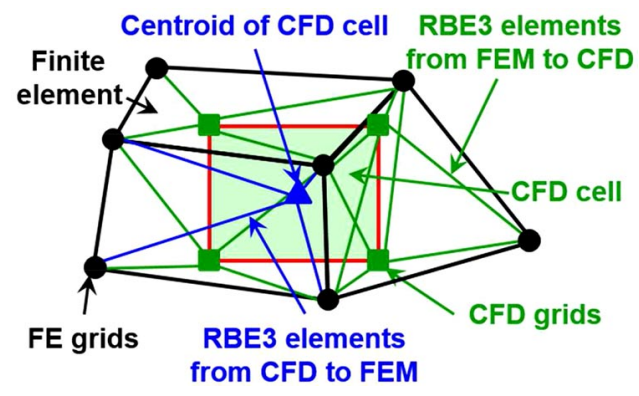

Fig. 6 RBE3 elements between structural grid points and CFD grids and centroids (FEM, finite element method). grid points and these centroids is also needed for the transient response computations with the MSC/NASTRAN code. In this study, RBE3 elements are also created between structural grids and these centroids of CFD cells, as shown in Fig. 6 . It should be noted that the well-known numerical problems associated with the Harder and Desmarais surface-spline technique [18] can be easily overcome through the use of the current technique with RBE3 elements.

The MSC/NASTRAN modal transient response analysis (solution 112) with 1024 time steps and a step size of $0.00060515 \mathrm{~s}$ is used to compute the strains, deflections, velocities, and accelerations. Time histories of aerodynamic force vectors at centroids of each CFD cell over the upper and lower wing surfaces are computed during unsteady CFL3D computation. These unsteady aerodynamic force vectors at each time step are converted to the applied force vectors at structural grids for the modal transient response analysis. The same initial velocity conditions used for the CFL3D computation are also used for the modal transient response analysis with the first three modes. The structural deflection and velocity values at the leading

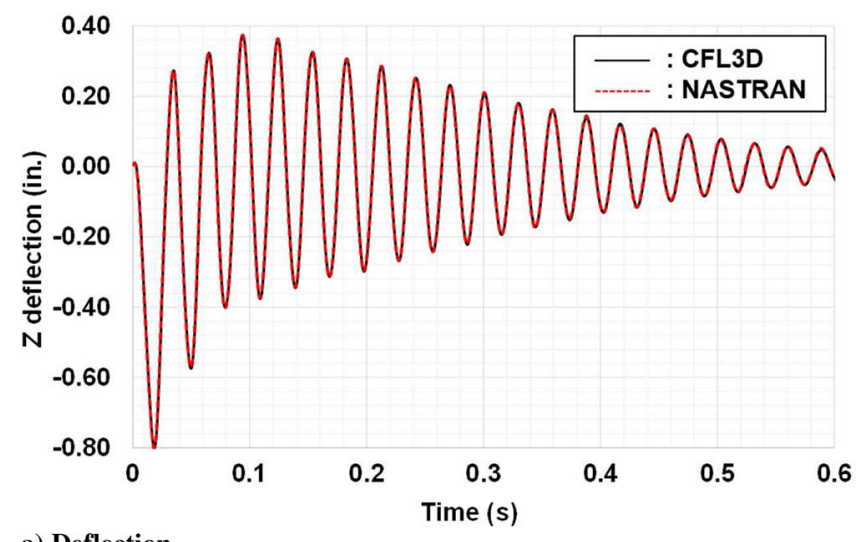

a) Deflection

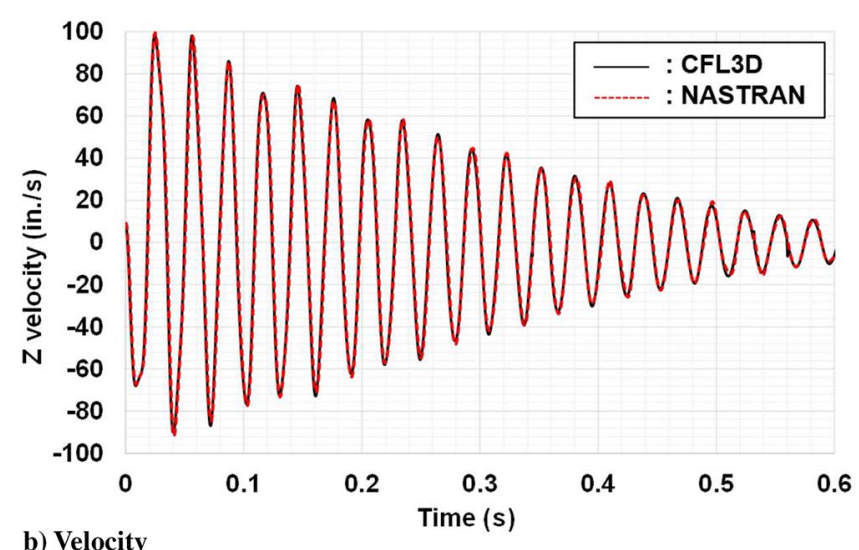

Fig. 7 Deflection and velocity comparisons using CFL3D and MSC/ NASTRAN codes. 

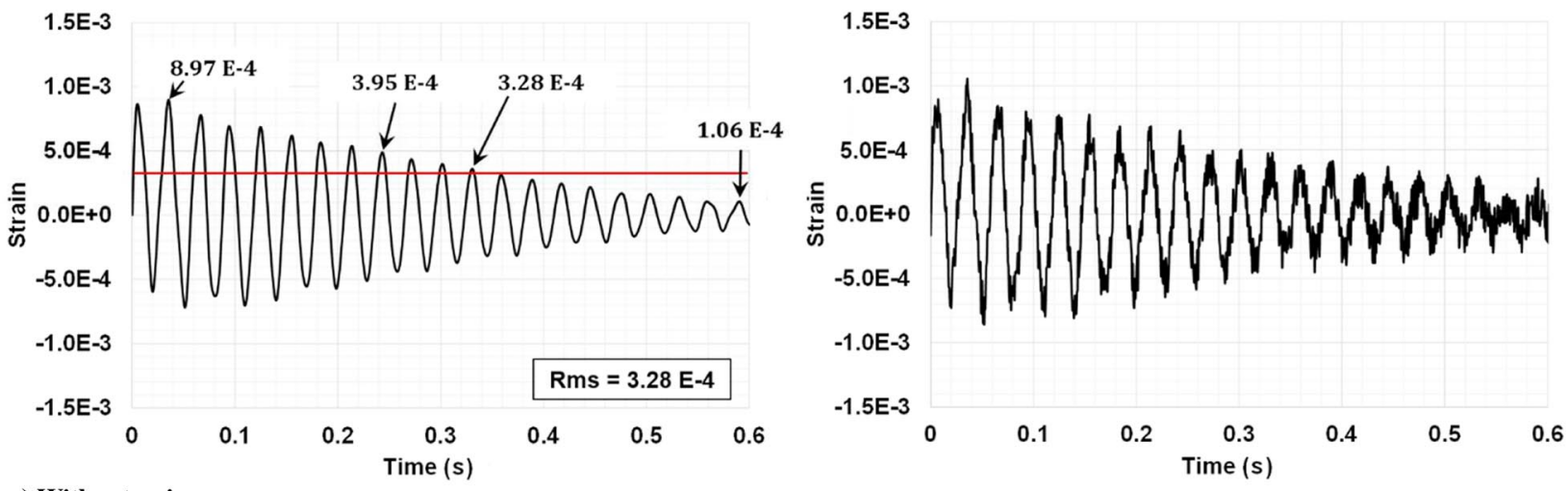

a) Without noise

b) $\mathrm{SNR}=10 \mathrm{~dB}$
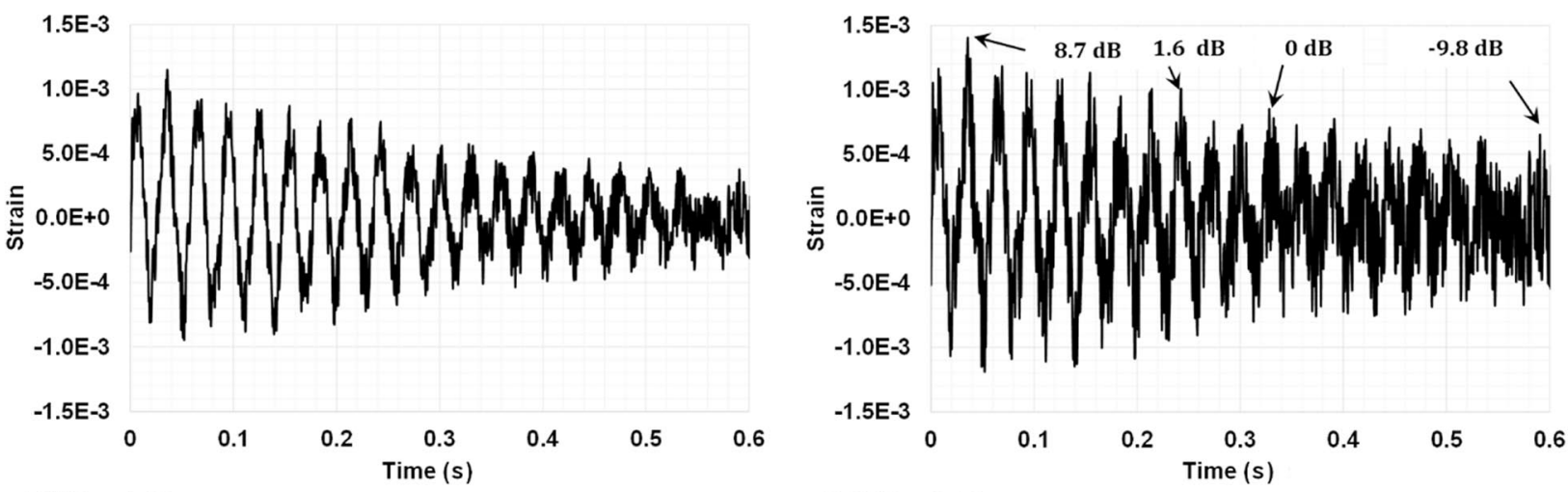

c) $\mathrm{SNR}=6 \mathrm{~dB}$

d) $\mathrm{SNR}=\mathbf{0} \mathrm{dB}$

Fig. 8 Time histories of strain under different levels of random white noise.

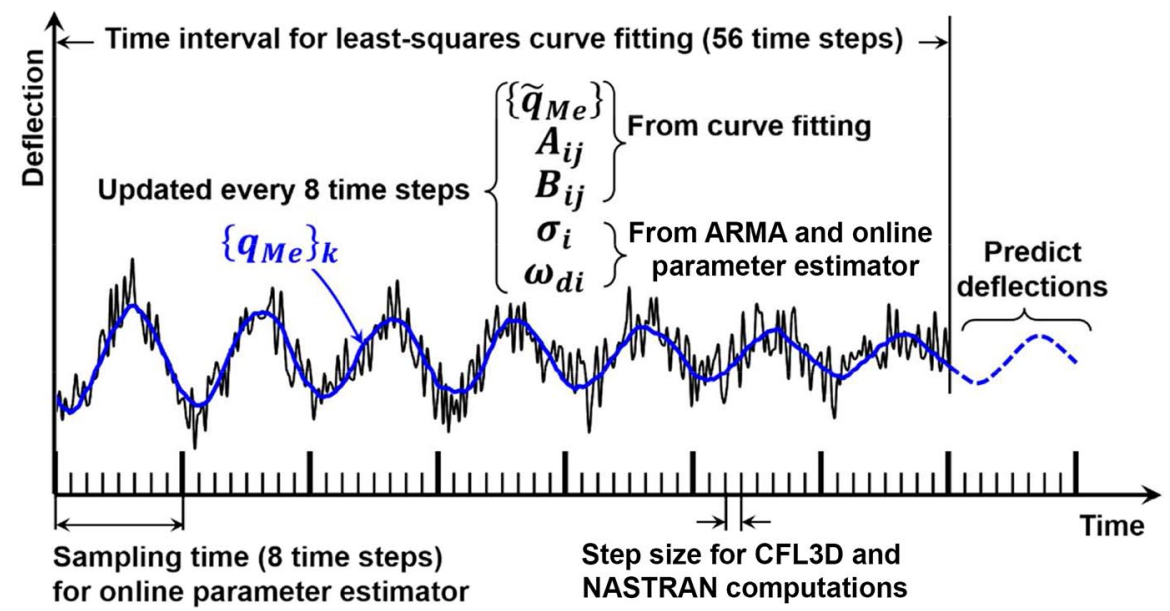

Fig. 9 Summary of the least-squares curve fitting and deflection prediction.

edge of the wingtip section obtained through the use of the CFL3D and MSC/NASTRAN codes are compared in Fig. 7. Excellent deflection and velocity matching are observed in this figure. Therefore, strain values computed from the MSC/NASTRAN code can be used as measured strain values to estimate the unsteady aerodynamic forces computed using the CFL3D computer simulation with the Euler equation.

Time histories of strain under different levels of random white noise are shown in Fig. 8. Figure 8a shows time histories of strain at the leading edge of the wing-root section. Random white noise is added to the unsteady strain data to demonstrate the robustness of the proposed approach. The strain signal-to-noise ratio (SNR) is defined as shown in Eq. (26):

$$
\mathrm{SNR} \equiv 20 \times \log _{10} \frac{\epsilon_{\mathrm{rms}}}{n_{\mathrm{rms}}}
$$

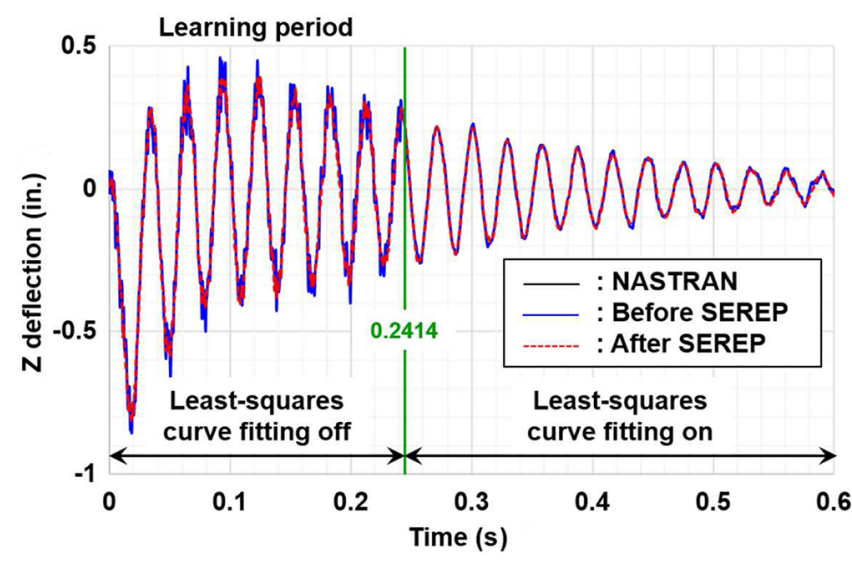

Fig. 10 Time histories of $Z$ deflection under $S N R=0 \mathrm{~dB}$. 


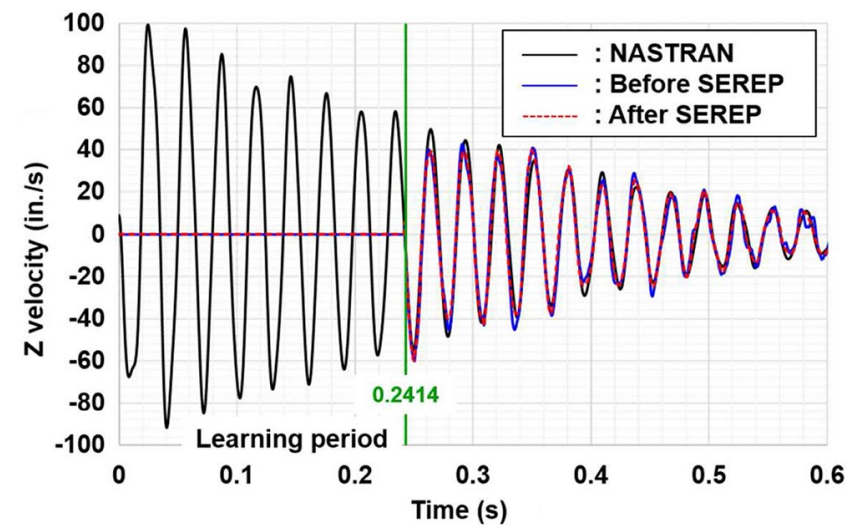

Fig. 11 Time histories of $Z$ velocity under $S N R=0 \mathrm{~dB}$.

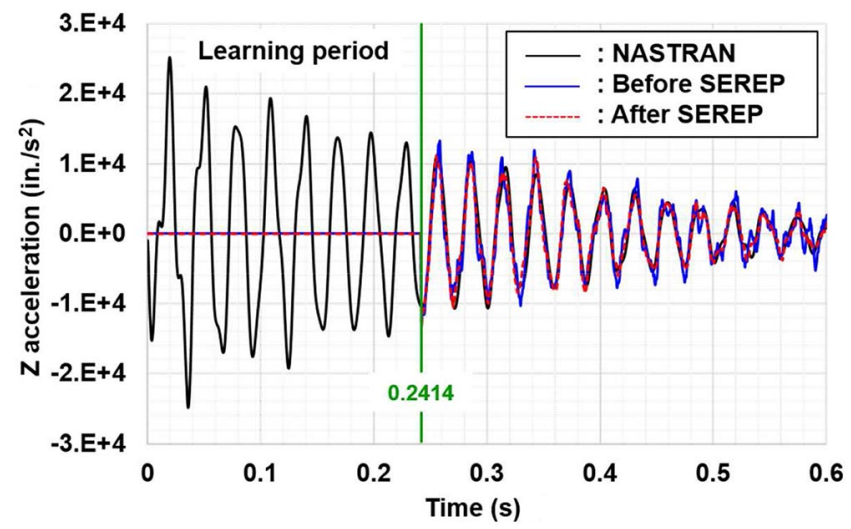

Fig. 12 Time histories of $Z$ acceleration under $S N R=0 \mathrm{~dB}$.

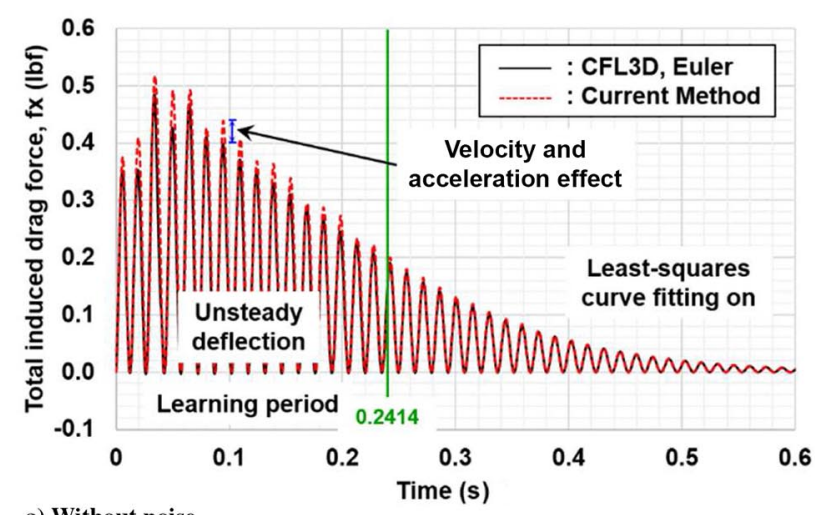

a) Without noise

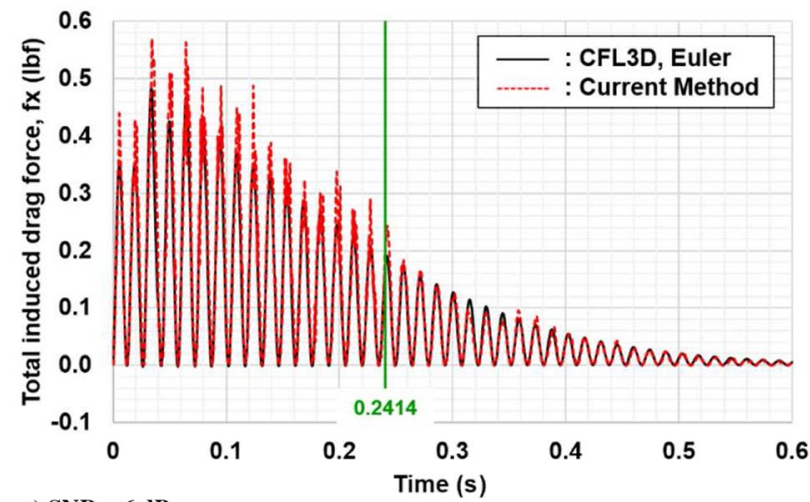

c) $\mathrm{SNR}=6 \mathrm{~dB}$ where $\epsilon_{\mathrm{rms}}$ and $n_{\mathrm{rms}}$ represent the root-mean-squared (rms) level of the unsteady strain and added noise, respectively. In this study, the SNRs of 10,6 , and $0 \mathrm{~dB}$ are used in the computer simulation. Corresponding time histories are shown in Figs. $\underline{\text { b }}-8 \mathrm{~d}$. The local SNR (LSNR) is defined as follows:

$$
\mathrm{LSNR} \equiv 20 \times \log _{10} \frac{\epsilon_{\mathrm{max}}}{n_{\mathrm{rms}}}
$$

where $\epsilon_{\max }$ is the local maximum absolute unsteady strain value. In Fig. 8d, the LSNR value is the same, with the SNR value near $0.33 \mathrm{~s}$. The $\overline{\mathrm{LSNR}}$ value is larger than the SNR value before $0.33 \mathrm{~s}$. The LSNR value becomes $-9.8 \mathrm{~dB}$ near $0.59 \mathrm{~s}$.

In this study, robustness of the proposed least-squares curve fitting method [Eq. (8)] is tested using time histories of unsteady strain, shown in Fig. $\underline{\overline{8}}$. A moving time window of 56 time steps is used in this curve fitting, as shown in Fig. 9. The least-squares curve fitting method in Eq. (8) is a nonlinear fitting problem; however, this nonlinear fitting problem becomes a linear problem when the damping factors and damped aeroelastic frequencies, $\sigma_{i}$ and $\omega_{d i}$, are provided. In this study, a sine Butterworth low-pass filter [15] with a cutoff frequency of $200 \mathrm{~Hz}$ is used to estimate reasonable frequencies and damping factors from unsteady strain data. The number of ARMA coefficients is seven, and the sampling time for this online estimator is $0.004236 \mathrm{~s}$ (eight steps). In this study, a recursive least-squares method based on Bierman's U-D factorization algorithm with a forgetting factor of 0.98 is used as an online parameter estimator [9]. Once the fitted coefficients $\left\{\tilde{\boldsymbol{q}}_{M e}\right\}$, $A_{i j}$, and $B_{i j}$ are obtained based on the current 56 time steps, then deflections are predicted for the next eight time steps. These eight steps correspond to the one sampling period for the online parameter estimator. As shown in Fig. 9, the damping factors and damped aeroelastic frequencies, $\sigma_{i}$ and $\bar{\omega}_{d i}$, are updated with every sampling time step.

Time histories of $Z$ deflection, velocity, and acceleration under $0 \mathrm{~dB}$ SNR are shown in Figs. 10-12, respectively. The least-squares curve fitting starts after the converged damping factors and damped

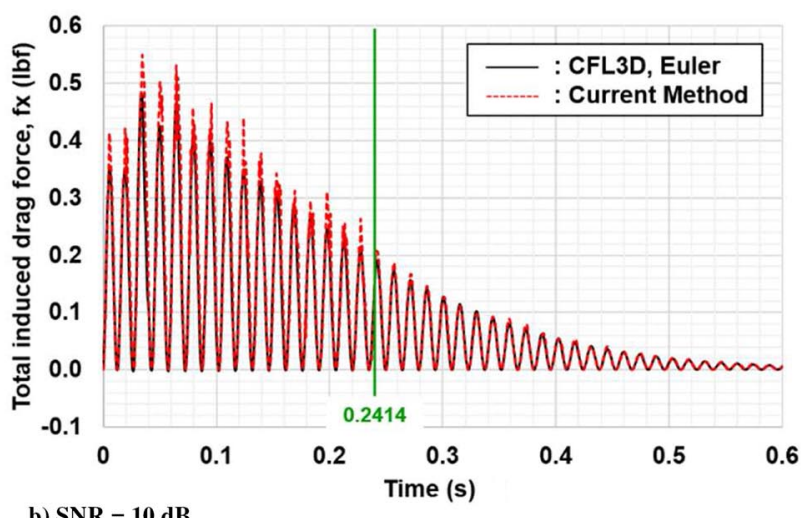

b) $\mathrm{SNR}=10 \mathrm{~dB}$

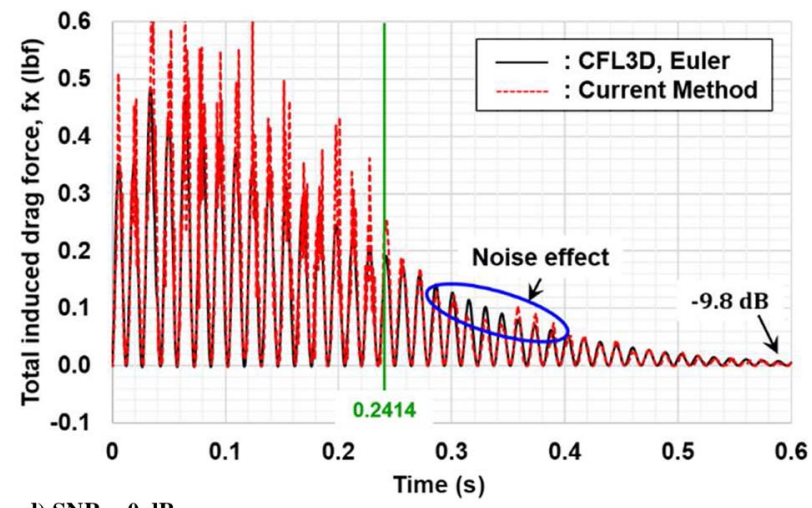

d) $\mathrm{SNR}=0 \mathrm{~dB}$

Fig. 13 Time histories of total induced drag force under different levels of random white noise. 
frequencies are obtained; thus, velocities and accelerations are not available until 400 steps (0.2414 s), as shown in Figs. 11 and 12. In Figs. $10-12$, the solid lines and dashed lines represent target values; and corresponding deflection, velocity, and acceleration values before $\left(\left\{\boldsymbol{q}_{M e}\right\}_{k},\left\{\dot{\boldsymbol{q}}_{M e}\right\}_{k}\right.$, and $\left.\left\{\ddot{\boldsymbol{q}}_{M e}\right\}_{k}\right)$ and after $\left(\{\boldsymbol{q}\}_{k},\{\dot{\boldsymbol{q}}\}_{k}\right.$, and $\left.\{\ddot{\boldsymbol{q}}\}_{k}\right)$ using the SEREP transformation, respectively.
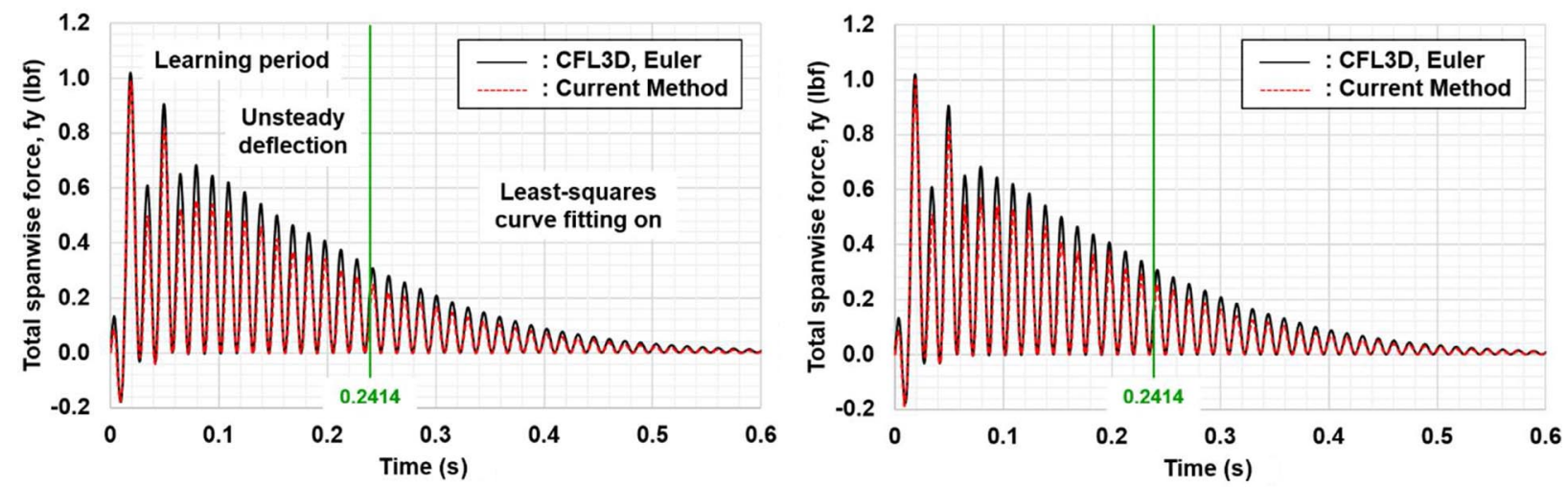

a) Without noise

b) $\mathrm{SNR}=10 \mathrm{~dB}$
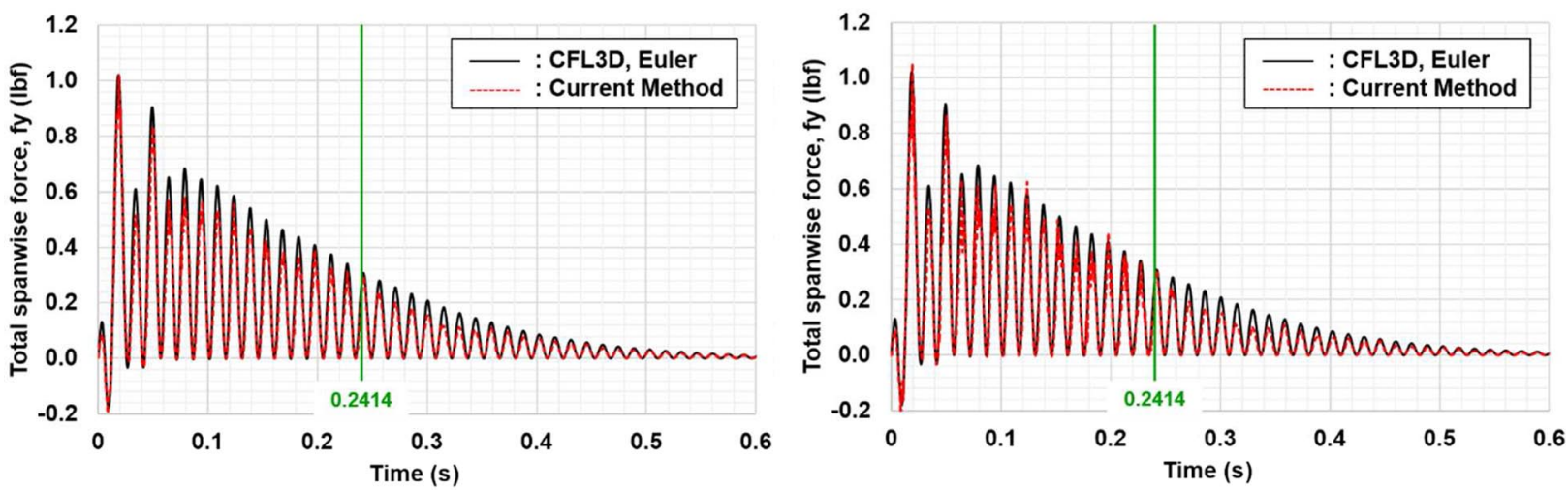

c) $S N R=6 \mathrm{~dB}$

d) $\mathrm{SNR}=0 \mathrm{~dB}$

Fig. 14 Time histories of total lateral force under different levels of random white noise.
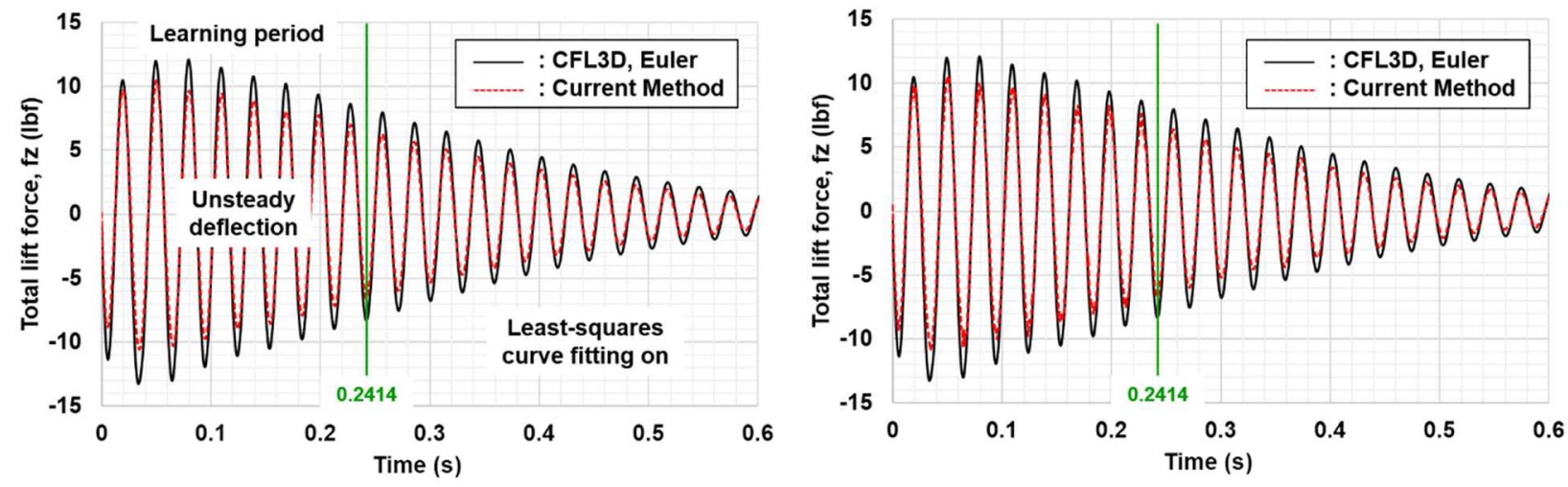

a) Without noise

b) $\mathrm{SNR}=10 \mathrm{~dB}$
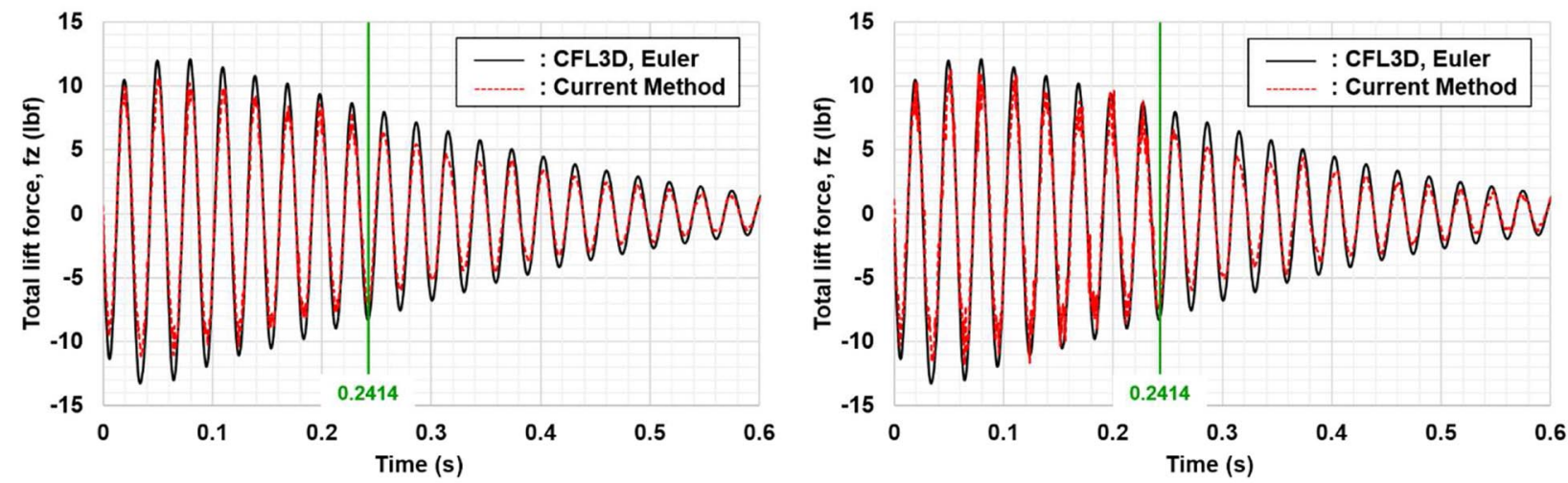

c) $\mathrm{SNR}=6 \mathrm{~dB}$

d) $\mathrm{SNR}=\mathbf{0} \mathrm{dB}$

Fig. 15 Time histories of total lift force under different levels of random white noise. 
The effect of the piecewise least-squares method for the computation of the unsteady deflection can be observed during the learning period as shown in Fig. 10. Even with noisy strain data (LSNR of 8.7 to $1.6 \mathrm{~dB}$ ), unsteady deflections are successfully obtained. The effect of the SEREP transformation can also be observed in Figs. 10-12 as the solid line versus the dashed line. Noise in the solid line becomes smoother after the SEREP transformation is applied. Finally, the effect of the least-squares curve fitting method in Eq. (8) can be clearly observed before and after $0.2414 \mathrm{~s}$, as shown in Fig. 10 . Noise in unsteady deflection during the learning period is drastically reduced after the least-squares curve fitting is used. Wing deflection, velocity, and acceleration are in excellent agreement with corresponding target values, as shown in Figs. 10-12. The proposed least-squares curve fitting method together with the analytical time derivatives performs excellently, even with an LSNR of $-9.8 \mathrm{~dB}$.

Modal AIC matrices are computed using the ZAERO code at Mach 0.714 . The ZAERO-based unsteady aerodynamic model configuration is shown in Fig. 2a. Reduced frequencies of 0.0, 0.006, 0.015, $0.035,0.08,0.13$, and $\overline{0.26}$ are selected for this computation. Roger's approximation with four aerodynamic lag terms is used for an RFA of these modal AIC matrices. The element-by-element least-squares curve fitting with a constraint at the steady-state condition, and a reduced frequency of zero, is used in the Roger's approximation procedure. Aerodynamics lag frequencies are $11.81 \mathrm{~Hz}$ $(\kappa=0.0177), 47.22 \mathrm{~Hz}(\kappa=0.0707), 106.2 \mathrm{~Hz}(\kappa=0.1591)$, and $188.9 \mathrm{~Hz}(\kappa=0.2829)$.

The total induced drag, lateral, and lift forces obtained from the current approach under different levels of random white noise are compared with the corresponding target aerodynamic forces from CFL3D computations in Figs. 13-15. The least-squares curve fitting method starts at $0.2414 \mathrm{~s}$ in Figs. 13-15. It is interesting that the computed forces between times of 0 to $0.2414 \mathrm{~s}$ are based on unsteady deflection only. Velocities and accelerations are assumed to be zero during the learning period, as shown in Figs. 11 and 12. The effects of noise can be observed in Fig. 13. Computed total induced drag forces with an SNR of $0 \mathrm{~dB}$ are the most noisy result, as shown in Fig. 13d.

The wing thickness effects on induced drag and lateral forces of 0.0353 and $0.0961 \mathrm{lbf}$, respectively, were subtracted from the CFD-based target force to have zero force at the steady-state condition in Figs. 13 and 14. In general, the current approach based on lifting-surface theory gave smaller forces than the target values in the cases of lift and lateral forces. The computed induced drag forces were in good agreement with the corresponding target drag force, as shown in Fig. 13.

Scaled total induced drag, and lateral and lift forces are shown in Fig. 16. In [19], unsteady aerodynamic model tuning of the aerostructures test wing 2 for accurate flutter prediction was performed at two different flight conditions, and the scaling factors obtained were 1.2579 and 1.2719. The average scaling factor of 1.2649 was multiplied to the aerodynamic forces. When this scaling factor was multiplied, the lateral and lift forces were in good agreement with the corresponding target values computed using the CFL3D code, as shown in Figs. $16 \mathrm{~b}$ and $16 \mathrm{c}$. Accuracy of the induced drag force in Fig. 16a became worse than the previous value. Therefore, it could be concluded that the small deviations between the current method and the CFL3D code with the Euler equation were mainly due to the uncertainty in the lifting-surface aerodynamic theory used in this study.

Recommendations for the practical application of unsteady deformation computations were given on page 1071 of [7]. First, it is recommended in this study that the lifting-surface theory used for the unsteady aerodynamic force computations be upgraded to a method based on steady and unsteady CFD computations to improve the accuracy of the current proposed approach with transonic aeroelasticity or high angle-of-attack flow. Second, an active induced drag control system based on the current proposed methodology will be a more physics-based approach than the drag control system based on measuring fuel flow. Finally, a reduced-order aeroelastic equation of motion with a smaller matrix size is recommended for an active

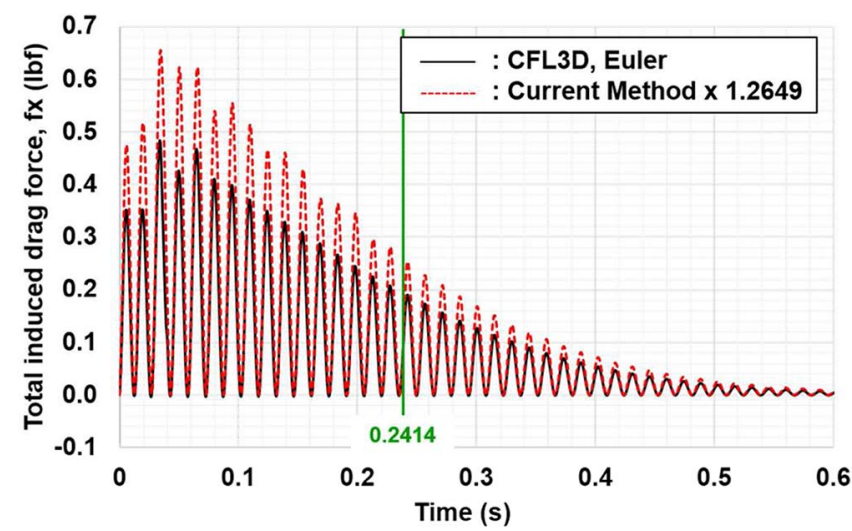

a) Scaled induced drag force

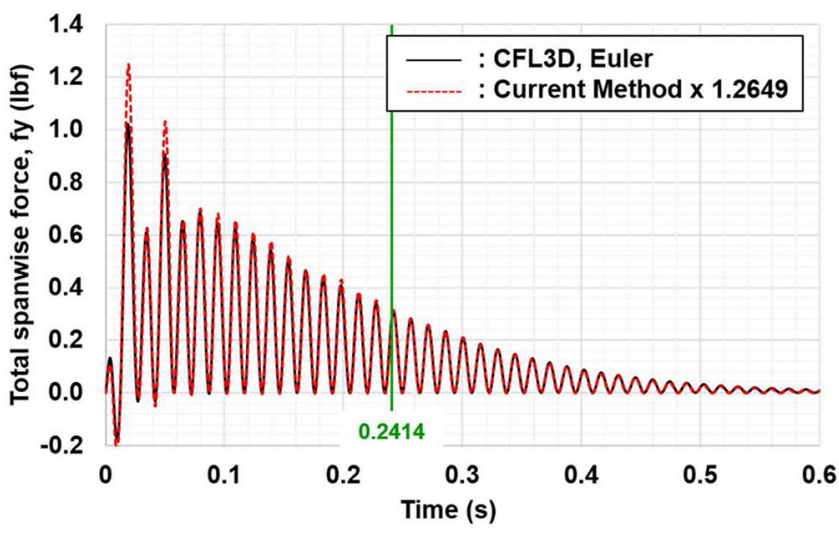

b) Scaled spanwise force

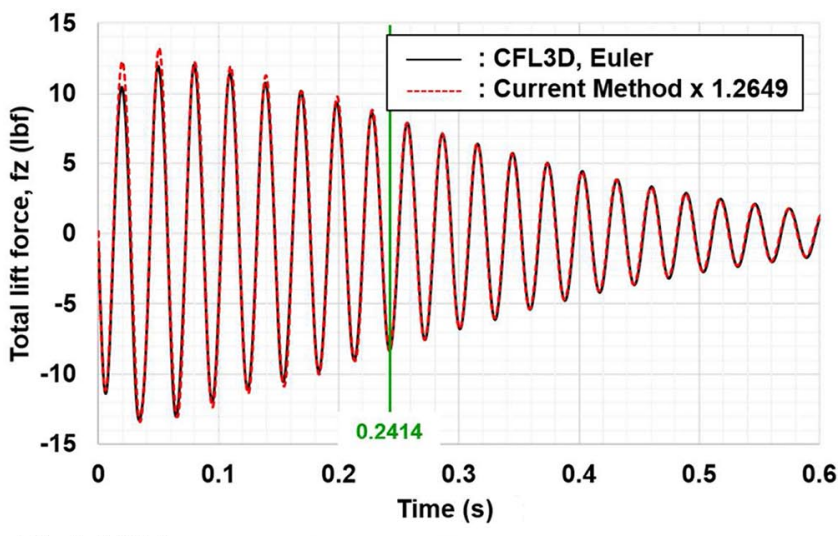

c) Scaled lift force

Fig. 16 Time histories of scaled total forces without white noise.

flexible motion control system, as well as an active induced drag control system.

\section{Conclusions}

In this study, unsteady aerodynamic forces were computed using simulated measured strain data. From unsteady strain information, unsteady structural deflections were computed using the two-step approach. Unsteady velocities and accelerations were computed using an autoregressive moving average model, an online parameter estimator, a low-pass filter, and a least-squares curve fitting method, together with analytical derivatives with respect to time. The deflections, velocities, and accelerations at each sensor location were independent of structural and aerodynamic models. The distributed strain data together with the current proposed approaches could therefore be used as distributed deflection, velocity, and acceleration sensors.

The general structural deflections, velocities, and accelerations were converted to the orthonormalized coordinates to compute 
orthonormalized aerodynamic force vectors using modal aerodynamic influence coefficient matrices. The modal aerodynamic influence coefficient matrices were fitted in the Laplace domain using Roger's approximation. Laplace-domain aerodynamics were converted to the time domain using a time-marching algorithm. The orthonormalized aerodynamic force vectors were transformed to the generalized coordinates using pseudomatrix inversion-based on singular-value decomposition. Finally, induced drag and lateral forces were obtained using surface-normal vectors. In general, computed aerodynamic forces based on the lifting-surface theory in subsonic speeds were in good agreement with the target aerodynamic forces generated using the CFL3D code with the Euler equation. This research demonstrated the feasibility of sensing induced drag and lift forces through the use of distributed sensor technology, together with the fiber-optic strain sensor. Thus, an active induced drag control system could be designed using these two computed aerodynamic forces, induced drag and lift, to improve the fuel efficiency of an aircraft.

In this study, interpolation elements (RBE3 elements in MSC/ NASTRAN terminology) between structural finite elements grids and the computational fluid dynamics grids and centroids were successfully incorporated with the unsteady aeroelastic computation scheme. The numerical problems often associated with the Harder and Desmarais surface-splines technique [18] are thus bypassed using the current technique with the RBE3 elements.

It should be emphasized that the deflection, velocity, and acceleration computation based on the proposed least-squares curve fitting method are validated with respect to the unsteady strain with a LSNR of $-9.8 \mathrm{~dB}$. Therefore, the current methodology of computing unsteady aerodynamic forces can be applied to the actual flight-test data. The most critical technology for the success of the proposed approach is the robust online parameter estimator because the leastsquares curve fitting method depends heavily on aeroelastic system frequencies and damping factors.

\section{References}

[1] Washburn, A., "Drag Reduction Status and Plans-Laminar Flow and AFC," AIAA Aerospace Sciences Meeting, Jan. 2011.

[2] Jahanmiri, M., "Aircraft Drag Reduction: An Overview," Dept. of Applied Mechanics, Chalmers Univ. of Technology, Research Rept. 2011:02, Göteborg, Sweden, 2011, p. 14.

[3] Ramakrishnan, M., Rajan, G., Semenova, Y., and Farrell, G., "Overview of Fiber Optic Sensor Technologies for Strain/Temperature Sensing Applications in Composite Materials," Sensors, Vol. 16, No. 99, 2016, Paper 99. doi: $10.3390 / \mathrm{s} 16010099$
[4] Igawa, H., Murayama, H., Nakamura, T., Yamaguchi, I., Kageyama, K., Uzawa, K., Wada, D., Ohsawa, I., Kanai, M., and Omichi, K., "Measurement of Distributed Strain and Load Identification Using $1500 \mathrm{~mm}$ Gauge Length FBG and Optical Frequency Domain Reflectometry," Proceedings of 20th International Conference on Optical Fiber Sensors, edited by J. Jones, B. Culshaw, W. Ecke, J. M. López-Higuera, and R. Willsch, Vol. 7503, 2009. doi: $10.1117 / 12.834236$

[5] Cusano, A., Cutolo, A., Nasser, J., Giordano, M., and Calabrò, A., "Dynamic Strain Measurements by Fibre Bragg Grating Sensor," Sensors and Actuators A: Physical, Vol. 110, Nos. 1-3, Feb. 2004, pp. 276-281. doi:10.1016/j.sna.2003.10.031

[6] Liu, T., Barrows, D. A., Burner, A. W., and Rhew, R. D., "Determining Aerodynamic Loads Based on Optical Deformation Measurements," AIAA Paper 2001-0560, 2001.

[7] Pak, C.-g., "Wing Shape Sensing from Measured Strain," AIAA Journal, Vol. 54, No. 3, 2016, pp. 1068-1077. doi:10.2514/1.J053986

[8] Pak, C.-g., and Truax, R. A., "Acceleration and Velocity Sensing from Measured Strain," AIAA Paper 2016-1229, 2016.

[9] Pak, C.-g., and Friedmann, P. P., "New Time Domain Technique for Flutter Boundary Identification," AIAA Paper 1992-2102, 1992.

[10] Bennett, R. M., and Desmarais, R. N., "Curve Fitting of Aeroelastic Transient Response Data with Exponential Functions," NASA Rept. SP-415, 1975.

[11] Krist, S. L., Biedron, R. T., and Rumsey, C. L., "CFL3D User's Manual (Version 5.0)," NASA Rept. TM-1998-208444, 1998.

[12] MSC/NASTRAN Quick Reference Guide Version 69, MacNeal Schwendler Corp., Los Angeles, CA, 1996.

[13] Pak, C.-g., Friedmann, P. P., and Livne, E., "Digital Adaptive Flutter Suppression and Simulation Using Approximate Transonic Aerodynamics," Journal of Vibration and Control, Vol. 1, No. 4, 1995, pp. 363-388. doi: $10.1177 / 107754639500100401$

[14] O'Callahan, J., Avitabile, P., and Riemer, R., "System Equivalent Reduction Expansion Process," Society for Experimental Mechanics, Union College, New York, 1989, pp. 29-37.

[15] Otnes, R. K., and Enochson, L., Applied Time Series Analysis: Basic Techniques, Vol. 1, Wiley, New York, 1978, pp. 421-422.

[16] Doggett, R. V., Jr., Rainey, A. G., and Morgan, H. G., "An Experimental Investigation of Aerodynamic Effects of Airfoil Thickness on Transonic Flutter Characteristics," NASA Rept. TMX-79, 1959.

[17] ZAERO User's Manual Version 8.2, ZONA Technology, Scottsdale, $\mathrm{AZ}, 2008$.

[18] Harder, R. L., and Desmarais, R. N., "Interpolation Using Surface Spines," Journal of Aircraft, Vol. 9, No. 2, 1972, pp. 189-191. doi: $10.2514 / 3.44330$

[19] Pak, C.-g., "Unsteady Aerodynamic Model Tuning for Precise Flutter Prediction," Journal of Aircraft, Vol. 48, No. 6, 2011, pp. 2178-2184. doi: $10.2514 / 1 . \mathrm{C} 031495$ 